\title{
SOME CHARACTERIZATIONS OF REGULAR MODULES
}

\author{
Goro AZumaYa
}

\begin{abstract}
Let $M$ be a left module over a ring $R . M$ is called a Zelmanowitz-regular module if for each $x \in M$ there exists a homomorphism $f: M \rightarrow R$ such that $f(x) x=x$. Let $Q$ be a left $R$-module and $h: Q \rightarrow M$ a homomorphism. We call $h$ locally split if for each $x \in M$ there exists a homomorphism $g: M \rightarrow Q$ such that $h(g(x))=x . M$ is called locally projective if every epimorphism onto $M$ is locally split. We prove that the following conditions are equivalent:

(1) $M$ is Zelmanowitz-regular.

(2) every homomorphism into $M$ is locally split.

(3) $M$ is locally projective and every cyclic submodule of $M$ is a direct summand of $M$.
\end{abstract}

As generalizations of the concept of Von Neumann's regular rings to the module case, there have been considered three types of modules by Fieldhouse [1], Ware [4] and Zelmanowitz [5], each called regular. The Fieldhouse-regular module was defined to be a module whose submodules are pure submodules and the Ware-regular module was defined as a projective module in which every cyclic submodule is a direct summand, while a left module $M$ over a ring $R$ is called a Zelmanowitz-regular module if for each $x \in M$ there exists a homomorphism $f: M \rightarrow R$ such that $f(x) x=x$. Now we introduce a notion of locally split homomorphisms to show that a module is Zelmanowitz. regular if and only if every homomorphism into the module is locally split, and by making use of this we prove that Zelmanowitz-regular modules are characterized as locally projective modules whose cyclic submodules are direct summands. For convenience (but at the risk of confusion), we call a module regular if every cyclic submodule of it is a direct summand. Thus, in this terminology, a module is Ware-regular or Zelmanowitz-regular if and only if it is projective regular or locally projective regular respectively. Moreover we shall see that every regular module is Fieldhouse-regular and that Ware-regular and Zelmanowitz-regular modules are also characterized as projective Fieldhouseregular and locally projective Fieldhouse-regular modules respectively.

Let $R$ be a ring with identity element. By a module we shall throughout mean a unital left $R$-module, unless otherwise specified. Let $Q$ and $M$ be modules, and let $h: Q \rightarrow M$ be a $(R-)$ homomorphism. $h$ is called locally split if for any $x_{\theta} \in h(Q)$ there exists a homomorphism $q: M \rightarrow Q$ such that $h\left(q\left(x_{0}\right)\right)=x_{0}$. 
Proposition 1. Let $h: Q \rightarrow M$ be a locally split homomorphism. Then, for any fnite number of $x_{1}, x_{2}, \ldots, x_{n} \in h(Q)$, there exists a homomorphism $q: M \rightarrow Q$ such that $h\left(q\left(x_{i}\right)\right)=x_{i}$ for $i=1,2, \ldots, n$.

Proof: In order to prove by induction, suppose that $n>1$ and our assertion is truc for $n-1$ (instead of $n$ ). Then there exists a $q_{1}: M \rightarrow Q$ such that $h\left(q_{1}\left(x_{i}\right)\right)=x_{i}$ for $i=1,2, \ldots, n-1$. Since $x_{n}-h\left(q_{1}\left(x_{n}\right)\right)$ is in $h(Q)$, there is a $q_{2}: M \rightarrow Q$ such that $h\left(q_{2}\left(x_{n}-h\left(q_{1}\left(x_{n}\right)\right)\right)\right)=x_{n}-h\left(q_{1}\left(x_{n}\right)\right)$. Let $q=q_{1}+q_{2}-q_{2} \circ h \circ q_{1}: M \rightarrow Q$. Then $h\left(q\left(x_{n}\right)\right)=h\left(q_{1}\left(x_{n}\right)\right)+h\left(q_{2}\left(x_{n}\right)\right)-$ $h\left(q_{2}\left(h\left(q_{1}\left(x_{n}\right)\right)\right)\right)=h\left(q_{1}\left(x_{n}\right)\right)+h\left(q_{2}\left(x_{n}-h\left(q_{1}\left(x_{n}\right)\right)\right)\right)=x_{n}$, and $h\left(q\left(x_{i}\right)\right)=$ $h\left(q_{1}\left(x_{i}\right)\right)+h\left(q_{2}\left(x_{i}\right)\right)-h\left(q_{2}\left(h\left(q_{1}\left(x_{i}\right)\right)\right)\right)=x_{i}+h\left(q_{2}\left(x_{i}\right)\right)-h\left(q_{2}\left(x_{i}\right)\right)=x_{i}$ for $i=1,2, \ldots, n-1$. Thus $q$ is a desired homomorphism.

Let $N$ be a submodule of a module $M . N$ is called locally split in $M$ if the inclusion map $N \rightarrow M$ is locally split, i.e., for any $x_{0} \in N$ therc exists a homomorphism $s: M \rightarrow N$ such that $s\left(x_{0}\right)=x_{0}$.

Proposition 2. Let $h: Q \rightarrow M$ be a homomorphism. Denote by $h^{\prime}$ the epimorphism $Q \rightarrow h(Q)$ regarded $h$ as a map onto $h(Q)$. Then $h$ is locally split if and only if $h^{\prime}$ is locally split and $h(Q)$ is locally split in $M$.

Proof: Let $x_{0}$ be any element of $h(Q)$. Suppose that $h$ is locally split. Then there exists a homomorphism $q: M \rightarrow Q$ such that $h\left(q\left(x_{0}\right)\right)=x_{0}$. This implies that the homomorphism $s=h \circ q: M \rightarrow h(Q)$ satisfies $s\left(x_{0}\right)=x_{0}$, and thus $h(Q)$ is locally split in $M$. On the other hand, if we denote by $q^{\prime}: h(Q) \rightarrow Q$ the restriction of $q$ to $h(Q)$ then we have $h^{\prime}\left(q^{\prime}\left(x_{0}\right)\right)=h\left(q\left(x_{0}\right)\right)=x_{0}$, which shows that $h^{\prime}$ is locally split. Suppose conversely that $h(Q)$ is locally split in $M$ and $h^{\prime}$ is also locally split. This means that there exist homomorphisms $s: M \rightarrow h(Q)$ and $q^{\prime}: h(Q) \rightarrow Q$ such that $s\left(x_{0}\right)=x_{0}$ and $h^{\prime}\left(q^{\prime}\left(x_{0}\right)\right)=x_{0}$. Let $q=q^{\prime} \circ s: M \rightarrow Q$. Then we have $h\left(q\left(x_{0}\right)\right)=h^{\prime}\left(q^{\prime}\left(s\left(x_{0}\right)\right)\right)=h^{\prime}\left(q^{\prime}\left(x_{0}\right)\right)=x_{0}$. Thus $h$ is locally split.

Proposition 3. Let $M$ be a module. Then every locally split submodule of $M$ is pure in $M$, while every locally split epimorphism from $M$ is pure, i.e., the kernel of the epimorphism is pure in $M$.

Proof: Let $N$ be a locally split submodule of $M$. Let $x_{1}, x_{2}, \ldots, x_{n} \in M$ satisfy the system of linear equations $r_{i 1} x_{1}+r_{i 2} x_{2}+\cdots+r_{i n} x_{n}=v_{i}(i=$ $1,2, \ldots, m$ ), where each $r_{i j} \in R$ and $v_{i} \in N$. Then, by applying Proposition 1 to $v_{1}, v_{2}, \ldots, v_{m}$ and the inclusion map $N \rightarrow M$ (instead of $x_{1}, x_{2}, \ldots, x_{n}$ and $h: Q \rightarrow M)$, we can find a homomorphism $s: M \rightarrow N$ such that $s\left(v_{i}\right)=v_{i}$ for $i=1,2, \ldots, m$. We have then $r_{i 1} s\left(x_{1}\right)+r_{i 2} s\left(x_{2}\right)+\cdots+r_{i n} s\left(x_{n}\right)=s\left(v_{i}\right)=$ $v_{i}(i=1,2, \ldots, m)$. Since each $s\left(x_{i}\right)$ is in $N$, this shows that $N$ is pure in $M$ by Cohn's theorem.

Let next $h: M \rightarrow M^{\prime}$ be an epimorphism and $N$ the kernel of $h$. Let $x_{1}, x_{2}, \ldots, x_{n} \in M$ satisfy the system of linear equations $r_{i 1} x_{1}+\tau_{i 2} x_{2}+\cdots+$ 
$r_{i n} x_{n}=v_{i}(i=1,2, \ldots, m)$, where $r_{i j} \in R$ and $v_{i} \in N$. Then we have $r_{i 1} h\left(x_{1}\right)+r_{i 2} h\left(x_{2}\right)+\cdots+r_{i n} h\left(x_{n}\right)=h\left(v_{i}\right)=0(i=1,2, \ldots, m)$. Suppose that $h$ is locally split. Then since each $h\left(x_{j}\right)$ is in $h(M)=M^{\prime}$, by applying Proposition 1 to $h\left(x_{1}\right), h\left(x_{2}\right), \ldots, h\left(x_{n}\right)$ and $h: M \rightarrow M^{\prime}$ (instead of $x_{1}, x_{2}, \ldots, x_{n}$ and $h: Q \rightarrow M)$, we find a homomorphism $q: M^{\prime} \rightarrow M$ such that $h\left(q\left(h\left(x_{j}\right)\right)\right)=$ $h\left(x_{j}\right)$, i.e., $x_{j}-q\left(h\left(x_{j}\right)\right) \in N$ for $j=1,2, \ldots, n$. From the above equalities it follows now $r_{i 1} q\left(h\left(x_{1}\right)\right)+r_{i 2} q\left(h\left(x_{2}\right)\right)+\cdots+r_{i n} q\left(h\left(x_{n}\right)\right)=0$ and therefore $r_{i 1}\left(x_{1}-q\left(h\left(x_{1}\right)\right)\right)+r_{i 2}\left(x_{2}-q\left(h\left(x_{2}\right)\right)+\cdots+r_{i n}\left(x_{n}-q\left(h\left(x_{n}\right)\right)=v_{i}(i=\right.\right.$ $1,2, \ldots, m)$. This implies that $N$ is pure in $M$ again by Cohn's theorem.

Remark. The notion of locally split submodules was introduced by Ramamurthi and Rangaswamy [2] by the name of strongly pure submodules, and they actually obtained the first half of the preceding proposition.

Theorem 4. Let $M$ be a left $R$-module. Then the following conditions are equivalent:

(1) $M$ is a Zelmanowitz-regular module.

(2) Every homomorphism into $M$ (from any module) is locally split.

(3) Every homomorphism $R \rightarrow M$ is locally split.

Proof: (1) $\Rightarrow(2)$ : Let $Q$ be a module and $h: Q \rightarrow M$ a homomorphism. Let $x_{0}$ be any element of $h(Q)$. Choose a $z_{0} \in Q$ such that $h\left(z_{0}\right)=x_{0}$. Since $M$ is Zelmanowitz-regular, there exists a homomorphism $f: M \rightarrow R$ such that $f\left(x_{0}\right) x_{0}=x_{0}$. Define a homomorphism $q: M \rightarrow Q$ by $q(x)=f(x) z_{0}$ for $x \in M$. Then we have $h\left(q\left(x_{0}\right)\right)=f\left(x_{0}\right) h\left(z_{0}\right)=f\left(x_{0}\right) x_{0}=x_{0}$, which shows that $h$ is locally split.

$(2) \Rightarrow(3)$ is trivial.

(3) $\Rightarrow(1)$ : Let $x_{0}$ be any element of $M$. Let $g: R \rightarrow M$ be the homomorphism defined by $g(r)=r x_{0}$ for $r \in R$. Then $g$ is locally split, so that there exists a homomorphism $f: M \rightarrow R$ such that $x_{0}=g\left(f\left(x_{0}\right)\right)=f\left(x_{0}\right) x_{0}$. This shows that $M$ is Zelmanowitz-regular.

Now we call a module $M$ a regular module if every submodule of $M$ is locally split in $M$.

Proposition 5. Let $M$ be a module. Then the following conditions are equivalent:

(1) $M$ is a regular module.

(2) Every finitely generated submodule of $M$ is a direct summand of $M$.

(3) Every cyclic submodule of $M$ is a direct summand of $M$.

Proof: (1) $\Rightarrow(2)$ : Let $N=R x_{1}+R x_{2}+\cdots+R x_{n}$ be a finitely generated submodule of $M$. Since $M$ is regular, $N$ is tocaliy split and therefore, by applying Proposition 1 to the inclusion map $N \rightarrow M$ (instead of $h: Q \rightarrow$ $M$ ), we can find a homomorphisin $s: M \rightarrow N$ such that $s\left(x_{i}\right)=x_{i}$ for $i=$ 
$1,2, \ldots, n$, or equivalently, $s(x)=x$ for all $x \in N$. This implies that $N$ is a direct summand of $M$.

(2) $\Rightarrow(3)$ is trivial.

(3) $\Rightarrow(1):$ Let $N$ be a submodule of $M$. Let $x_{0}$ be any element of $N$. Then the cyclic submodule $R x_{0}$ is a direct summand of $M$, which means that there is a homomorphism $s: M \rightarrow R x_{0}(\subset N)$ such that $s\left(x_{0}\right)=x_{0}$. Thus $N$ is locally split in $M$.

It is to be pointed out that every submodule of a regular module is regular too, and every regular module is Fieldhouse-regular, i.e., every submodule is a pure submodule.

A module $M$ is called locally projective if every epimorphism onto $M$ (from any module) is locally split. It follows from Proposition 3 that every locally projective module is fiat, since a flat module is characterized as a module onto which every epimorphism is pure. The notion of locally projective modules was introduced by Zimmermann-Huisgen [6] and also by Raynaud and Gruson [3] under the name of flat strict Mittag-Leffer modules. Their definitions are apparently different from the above one. But the following proposition implies that all the definitions coincide (if compared with [6], Theorem 2.1 and [3], Proposition 2.3.4), and we will give a proof to the proposition for completeness:

Proposition 6. Let $M$ be a left $R$-module. Then the following conditions are equivalent:

(1) $M$ is locally projective.

(2) For any finitely generated submodule $M_{0}$ of $M$, there exist a finitely generated free left $R$-module $F$ aand homomorphisms $f: M \rightarrow F$ and $g: F \rightarrow$ $M$ such that $g(f(x))=x$ for all $x \in M_{0}$.

(9) For any $x_{0} \in M$, there exist a finite number of homomorphisms $f_{i}$ : $M \rightarrow R(i=1,2, \ldots, n)$ and elements $y_{i} \in M(i=1,2, \ldots, n)$ such that $f_{1}\left(x_{0}\right) y_{1}+f_{2}\left(x_{0}\right) y_{2}+\cdots+f_{n}\left(x_{0}\right) y_{n}=x_{0}$.

Proof: (1) $\Rightarrow(2)$ : Let $Q$ be a free $R$-module having an epirnorphism $h$ : $Q \rightarrow M$. Then $h$ is locally split, so that, by applying Proposition 1 to the finite number of generators of $M_{0}$, we can find a homomorphism $q: M \rightarrow Q$ such that $h(q(x))=x$ for all $x \in M_{0}$. Since the image $q\left(M_{0}\right)$ of $M_{0}$ is a finitely generated submodule of $Q$, there exists a finite subset $\left\{u_{1}, u_{2}, \ldots, u_{n}\right\}$ of the free basis of $Q$ such that $q\left(M_{0}\right)$ is contained in the finitely generated free submodule $F=R u_{1}+R u_{2}+\cdots+R u_{n}$ of $Q$. Since $F$ is a direct summand of $Q$, there exists a homomorphism $p: Q \rightarrow F$ such that $p(z)=z$ for all $z \in F$. Let $f=p \circ q: M \rightarrow F$, and let $g: F \rightarrow M$ be the restriction of $h$ to $F$. Then they clearly satisfy $g(f(x))=x$ for all $x \in M_{0}$.

(2) $\Rightarrow(3)$ : Let $x_{0} \in M$. Since $R x_{0}$ is finitely generated, there exist a finitely generated free $R$-module $F$ and homomorphisms $f: M \rightarrow F, g: F \rightarrow M$ such that $g\left(f\left(x_{0}\right)\right)=x_{0}$. Let $u_{1}, u_{2}, \ldots, u_{n}$ be a free basis of $F$. Then we can, for 
each $i$, define a homomorphism $f_{1}: M \rightarrow R$ by $f(x)=f_{1}(x) u_{1}+f_{2}(x) u_{2}+$ $\ldots+f_{n}(x) u_{n}$ for $x \in M$. Let $y_{i}=g\left(u_{i}\right) \in M$ for $i=1,2, \ldots, n$. Then we have $x_{0}=g\left(f\left(x_{0}\right)\right)=f_{1}\left(x_{0}\right) y_{1}+f_{2}\left(x_{0}\right) y_{2}+\cdots+f_{n}\left(x_{0}\right) y_{n}$.

(3) $\Rightarrow(1)$ : Let $Q$ be any $R$-module having an epimorphism $h: Q \rightarrow M$. Let $x_{0} \in M$, and let $f_{i}: M \rightarrow R$ and $y_{i} \in M(i=1,2, \ldots, n)$ be as in (3). Let $z_{i} \in Q$ be such that $h\left(z_{i}\right)=y_{i}$ for each $i$, and define a homomorphism $q: M \rightarrow Q$ by $q(x)=f_{1}(x) z_{1}+f_{2}(x) z_{2}+\cdots+f_{n}(x) z_{n}$ for $x \in M$. Then we have that $h\left(q\left(x_{0}\right)\right)=f_{1}\left(x_{0}\right) y_{1}+f_{2}\left(x_{0}\right) y_{2}+\cdots+f_{n}\left(x_{0}\right) y_{n}=x_{0}$. Thus $h$ is locally split, so that $M$ is locally projective.

Proposition 7. Let $M$ be a locally projective module, and let $N$ be a pure submodule of $M$. Then $N$ is locally projective and is locally split in $M$.

Proof: Let $x_{0}$ be any element of $N$. By the preceding proposition, there exist homomorphisms $f_{i}: M \rightarrow R$ and elements $y_{i} \in M(i=1,2, \ldots, n)$ such that $f_{1}\left(x_{0}\right) y_{1}+f_{2}\left(x_{0}\right) y_{2}+\cdots+f_{n}\left(x_{0}\right) y_{n}=x_{0}$. Since $N$ is pure in $M$, we can find elements $v_{1}, v_{2}, \ldots, v_{n}$ in $N$ such that $f_{1}\left(x_{0}\right) v_{1}+f_{2}\left(x_{0}\right) v_{2}+\cdots+f_{n}\left(x_{0}\right) v_{n}=x_{0}$ according to Cohn's criterion. Now we define a homomorphism $s: M \rightarrow N$ by $s(x)=f_{1}(x) v_{1}+f_{2}(x) v_{2}+\cdots+f_{n}(x) v_{n}$ for $x \in M$. Then we have that $s\left(x_{0}\right)=x_{0}$. Thus $N$ is locally split in $M$. On the other hand, if we denote by $g_{i}$ the restriction of $f_{i}$ to $N$ then clearly we have that $g_{1}\left(x_{0}\right) v_{1}+g_{2}\left(x_{0}\right) v_{2}+$ $\cdots+g_{n}\left(x_{0}\right) v_{n}=x_{0}$, which shows that $N$ is locally projective.

Remark. That $N$ is locally projective in Proposition 7 was mentioned in [6, p. 236].

Theorem 8. Let $M$ be a module. Then the following conditions are equivalent:

(1) $M$ is a Zelmanowitz-regular module.

(2) $M$ is a locally projective regular module.

(3) $M$ is locally projective and Fieldhouse-regular (i.e., every submodule of $M$ is pure in $M$ ).

Proof: (1) $\Rightarrow(2)$ : If $M$ is Zelmanowitz-regular, it follows from Theorem 4 that every epimorphism onto $M$ is locally split and every monomorphism into $M$ is locally split, which mean that $M$ is locally projective and regular respectively. (Another proof for the local projectivity of $M$ can be obtained directly from Proposition 6 , for that for any $x_{0} \in M$ there exists an homomorphism $f: M \rightarrow R$ such that $f\left(x_{0}\right) x_{0}=x_{0}$ implies that $M$ satisfies the condition (3) in Proposition 6 with $n=1, f_{1}=f$ and $y_{1}=x_{0}$. That a Zelmanowitzregular module is regular, i.e., every cyclic submodule of the module is a direct summand, is also proved in [5, Theorem 1.6].

$(2) \Rightarrow(3)$ is a consequence of the fact, due to Proposition 3, that every locally split submodule is a pure submodule. 
(3) $\Rightarrow$ (1) : Let $Q$ be a module and $h: Q \rightarrow M$ a homomorphism. Since $h(Q)$ is a pure submodule of $M$ by assumption, it follows from Proposition 7 that $h(Q)$ is locally projective and is locally split in $M$. Regarding $h$ as a map onto $h(Q)$ we have an epimorphism $h^{\prime}: M \rightarrow h(Q)$, but the local projectivity of $h(Q)$ implies that $h^{\prime}$ is locally split. Therefore, by Proposition $2, h$ is locally split. Thus, $M$ is Zelmanowitz-regular according to Theorem 4.

Remark 1. Although we throughout assume that $R$ has an identity element, the paper [5] deals with modules over rings without identity element.

Remark 2. It is pointed out in [6] that over a regular ring a module is locally projective if and only if it is Zelmanowitz-regular. But this can be regarded as a particular case of Theorem 8 , because over a regular ring every module is flat and hence is Fieldhouse-regular.

In this connection, we would like to mention of some properties of regular modules and locally projective modules:

1. If $M$ is a regular $R$-module then its Jacobson radical $J(M)$ is zero, and if $M$ is a faithful regular $R$-module then the Jacobson radical $J(R)$ of $R$ is zero.

The proof is actually given in [4], though regular modules in [4] mean projective regular modules in the present paper. Namely, if $x_{0}$ is in $J(M)$ then $R x_{0}$ is a direct summand small submodule of $M$ and therefore $x_{0}=0$, which implies $J(M)=0$. Since $J(R) M \subset J(M)$, it follows $J(R)=0$ if $M$ is faithful and regular.

2. If $M$ is a locally projective $R$-module then $J(R) M=J(M)$.

For, let $x_{0}$ be in $J(M)$; then by Proposition 6 there exist a finite number of homomorphisms $f_{i}: M \rightarrow R$ and elements $y_{i}$ in $M(i=1,2, \ldots, n)$ such that $f_{1}\left(x_{0}\right) y_{1}+f_{2}\left(x_{0}\right) y_{2}+\cdots+f_{n}\left(x_{0}\right) y_{n}=x_{0}$. Let $L$ be a maximal left ideal of $R$. Then its inverse image by $f_{i}$ is either equal to $M$ or a maximal submodule of $M$ and therefore contains $J(M)$, or equivalently, $f_{i}(J(M)) \subset L$. Since this is true for every maximal left ideal $L$, it follows $f_{i}(J(M)) \subset J(R)$ and in particular $f_{i}\left(x_{0}\right) \in J(R)$. This is true for each $i=1,2, \ldots, n$, so that we have $x_{0} \in J(R) M$. Thus we know that $J(M) \subset J(R) M$.

3. A module $M$ is Fieldhouse-regular if (and only if) every finitely generated submodule of $M$ is pure in $M$.

This is because Cohn's criterion for purity is concerned only with finite number of elements.

Proposition 9. Let $M$ be a Zelmanowitz-regular module, and let $S$ be the endomorphism ring of $M$. Then, as an $S$-module, $M$ is Zelmanowitz-regular too, and the Jacobson radical $J(S)$ of $S$ is zero.

Proof: We consider $M$ a right $S$-module and hence a two-sided $R$ - $S$-module; thus $s t=t \circ s$ for all $s, t \in S$. Let $x_{0}$ be an element of $M$. Then there exists a homomorphism $f: M \rightarrow R$ such that $f\left(x_{0}\right) x_{0}=x_{0}$. Let $y \in M$. Then the mapping $x \mapsto f(x) y$ for $x \in M$ is an endomorphism of $M$, which we denote by $\bar{y} \in S$. If $s \in S$, we have $f(x)(y s)=(f(x) y) s$ for all $x \in M$, i.e., $\overline{y s}=\bar{y} s$. 
This implies that the mapping $y \mapsto \bar{y}$ for $y \in M$ is a homomorphism $M \rightarrow S$ as $S$-modules. If we denote this by $g$ then we have $f(x) y=x g(y)$ for all $x$, $y \in M$. (In the notation in [5], $g(y)=[f, y]$ for all $y \in M$.) It follows in particular that $x_{0}=f\left(x_{0}\right) x_{0}=x_{0} g\left(x_{0}\right)$. This shows that the $S$-module $M$ is Zelmanowitz-regular. Since $M$ is a faithful $S$-module, we have $J(S)=0$ according to the above mentioned property 1.

Now, clearly a locally projective module is projective if it is finitely generated, but this is true even if it is countably generated:

Proposition 10. Every countably generated locally projective module is projective.

Proof: If we observe the fact that every locally projective module is a MittagLeffler module, our proposition can be regarded as a particular case of [3], Corollaire 2.2.2. But we shall for completeness give a proof which is valid for our case. Let $M$ be a locally projective $R$-module with countable generators $x_{1}, x_{2}, x_{3}, \ldots$. Let $M_{1}=R x_{1}$. By Proposition 6 there exist a finitely generated free $R$-module $F_{1}$ and homomorphisms $f_{1}: M \rightarrow F_{1}, g_{1}: F_{1} \rightarrow M$ such that $g_{1}\left(f_{1}(x)\right)=x$ for all $x \in M_{1}$. Let next $M_{2}=g_{1}\left(F_{1}\right)+R x_{2}$. Since $M_{2}$ is finitely generated, again by Proposition 6 , there exist a finitely generated free $R$-module $F_{2}$ and homomorphisms $f_{2}: M \rightarrow F_{2}, g_{2}: F_{2} \rightarrow M$ such that $g_{2}\left(f_{2}(x)\right)=x$ for all $x \in M_{2}$. In this way, for each $n>1$, we can find a finitely generated free $R$-module $F_{n}$ and homomorphisms $f_{n}: M \rightarrow F_{n}, g_{n}: F_{n} \rightarrow M$ such that $g_{n}\left(f_{n}(x)\right)=x$ for all $x \in M_{n}=g_{n-1}\left(F_{n-1}\right)+R x_{n}$. But this is clearly equivalent to that $g_{n}\left(f_{n}\left(g_{n-1}(y)\right)\right)=g_{n-1}(y)$ for all $y \in F_{n-1}$ and $g_{n}\left(f_{n}\left(x_{n}\right)\right)=x_{n}$. From this follows then that $g_{n} \circ f_{n} \circ g_{n-1}=g_{n-1}$ whence $g_{n-1}\left(F_{n-1}\right) \subset g_{n}\left(F_{n}\right)$ and $x_{n} \in g_{n}\left(F_{n}\right)$. Thus we have an ascending chain $g_{1}\left(F_{1}\right) \subset g_{2}\left(F_{2}\right) \subset g_{3}\left(F_{3}\right) \subset \ldots$ of submodules of $M$ whose union is equal to $M$. For simplicity, we put $s_{n}=g_{n} \circ f_{n}: M \rightarrow g_{n}\left(F_{n}\right)$ for each $n$. Then $s$ is an endomorphism of $M$ satisfying $s_{n} \circ g_{n-1}=g_{n-1}$ and hence $s_{n} \circ s_{n-1}=s_{n-1}$ for each $n>1$. Moreover we point out that $s_{n} \circ g_{r}=g_{r}$ and $s_{n} \circ s_{r}=s_{r}$ whenever $n>r$, because if $r<n$ then $g_{r}\left(F_{r}\right) \subset g_{n-1}\left(F_{n-1}\right)$ and so $s_{n}\left(g_{r}(y)\right)=g_{r}(y)$ for all $y \in F_{r}$.

Let $F$ be the direct sum of all $F_{n}$ 's. Then $F$ is a countably generated free $R$ module. The homomorphisms $g_{n}: F_{n} \rightarrow M$ for $n=1,2,3, \ldots$ together define a homomorphism $g: F \rightarrow M$ in the natural manner. The image $g(F)$ is the sum of all $g_{n}\left(F_{n}\right)^{\prime} s$ and hence is equal to $M$, because even their union is $M$. Thus $g$ is an epimorphism. In order to prove that $M$ is projective, it is therefore sufficient to show that $g$ splits, i.e., there exists a homomorphism $f: M \rightarrow F$ such that $g \circ f=1$, the identity map of $M$. Let now $q_{n}: F_{n} \rightarrow F$ be the canonical embedding for $n=1,2,3, \ldots$ Then we have $g \circ q_{n}=g_{n}$ for each $n$. We shall construct a homomorphism $h_{n}: F_{n} \rightarrow F$ for each $n$ such that $g \circ h_{n}=g_{n}$ and $h_{n} \circ f_{n} \circ g_{n-1}=h_{n+1} \circ f_{n+1} \circ g_{n-1}$ if $n>1$. For this purpose, let first $h_{1}=q_{1}$. Then $g \circ h_{1}=g_{1}$. Suppose $n>1$ and there is given an $h_{n}: F_{n} \rightarrow F$ such 
that $g \circ h_{n}=g_{n}$. We define $h_{n+1}=\left(h_{n} \circ f_{n}+q_{n+2} \circ f_{n+2} \circ\left(1-s_{n}\right)\right) \circ g_{n+1}$. Then we have $g \circ h_{n+1}=\left(g \circ h_{n} \circ f_{n}+g \circ q_{n+2} \circ f_{n+2} \circ\left(1-s_{n}\right)\right) \circ g_{n+1}=$ $\left(g_{n} \circ f_{n}+g_{n+2} \circ f_{n-2} \circ\left(1-s_{n}\right)\right) \circ g_{n+1}=\left(s_{n}+s_{n+2} \circ\left(1-s_{n}\right)\right) \circ g_{n+1}=$ $\left(s_{n}+s_{n+2}-s_{n+2} \circ s_{n}\right) \circ g_{n+1}=s_{n+2} \circ g_{n+1}=g_{n+1}$. On the other hand, we have $h_{n+1} \circ f_{n+1} \circ g_{n-1}=\left(h_{n} \circ f_{n}+q_{n+2} \circ f_{n+2} \circ\left(1-s_{n}\right)\right) \circ s_{n+1} \circ g_{n-1}=$ $\left(h_{n} \circ f_{n}+q_{n+2} \circ f_{n+2} \circ\left(1-s_{n}\right)\right)_{\circ} g_{n} n-1=h_{n} \circ f_{n} \circ g_{n-1}+q_{n-2} \circ f_{n-2} \circ g_{n-1}-$ $q_{n-2} \circ f_{n-2} \circ s_{n} \circ g_{n-1}=h_{n} \circ f_{n} \circ g_{n-1}$. Thus by induction we get a desired sequence of homomorphisms $h_{n}(n=1,2,3, \ldots)$.

Let $x \in M$. Then there exists an $n>1$ such that $x \in g_{n-1}\left(F_{n-1}\right)$ i.e., $x=$ $g_{n-1}(y)$ for some $y \in F_{n-1}$. We have then that $h_{n}\left(f_{n}(x)\right)=h_{n}\left(f_{n}\left(g_{n-1}(y)\right)\right)=$ $h_{n+1}\left(f_{n+1}\left(g_{n-1}(y)\right)\right)=h_{n+1}\left(f_{n+1}(x)\right)$. Moreover, since $x \in g_{n}\left(F_{n}\right)$ in this case, by replacing $n$ by $n+1$ we should have that $h_{n+1}\left(f_{n+1}(x)\right)=h_{n+2}\left(f_{n+2}(x)\right)$. Continuing in this way, we confirm that $h_{n}\left(f_{n}(x)\right)=h_{m}\left(f_{m}(x)\right)$ for cvery $m>n$. This shows that $h_{n}\left(f_{n}(x)\right)$ is independent of the choice of $n$ so far as $x$ is in $g_{n-1}\left(F_{n-1}\right)$. Thus by defining $f(x)=h_{n}\left(f_{n}(x)\right)$ for $x \in M$ we have a homomorphism $f: M \rightarrow F$, which satisfies $g(f(x))=g_{n}\left(f_{n}(x)\right)=x$ (since $\left.x \in g_{n-1}\left(F_{n-1}\right)\right)$. This completes our proof.

It is to be pointed out that the preceding proposition.can be regarded as a generalization of [5, Corollary 1.7.].

Acknowledgments. The author gratefully acknowledges the support from Institut d'Estudis Catalans of Barcelona.

\section{References}

1. D.J. Fieldhouse, Pure theories, Math. Ann 184 (1969), 1-18.

2. V.S. RAMAMURTHI AND K.M. RANGASWAMY, On finitely injective modules, J. Austral Math. Soc. 16 (1973), 239-248.

3. M. Raynaud and L. Gruson, Critères de platitude et de projective, Invent. Math. 13 (1971), 1-89.

4. R. WAnE, Endomorphism rings of projective modules, Thans. Amer. Math. Soc. 155 (1971), 233-259.

5. J. Zflmanowitz, Regular modules, Trans. Amer. Math. Soc. 163 (1972), $341-355$.

6. B. ZIMMERMANN-HuISGEN, Pure submodules of direct products of free modules, Math. Ann. 224 (1976), 233-245.

\footnotetext{
Department of Mathematics

Indiana University

Bloomington, IN 4740 s

U.S.A.
}

Rebut el 2 de Juny de 1989 